\title{
Advances in the role of exosomal non-coding RNA in the development, diagnosis, and treatment of gastric cancer (Review)
}

\author{
PENG-FEI GAO*, DA HUANG* , JUN-YAN WEN, WEI LIU and HONG-WU ZHANG \\ Department of Anatomy, Guangdong Provincial Key Laboratory of Construction and Detection in Tissue Engineering, \\ Southern Medical University, Guangzhou, Guangdong 510515, P.R. China
}

Received February 9, 2020; Accepted May 15, 2020

DOI: $10.3892 / \mathrm{mco} .2020 .2068$

\begin{abstract}
Exosomes are small vesicles secreted by a variety of cells that contain vrious biological macromolecules, including RNA, non-coding RNA and protein. An increasing number of studies have demonstrated that exosomes and particularly the non-coding RNAs they contain, serve important roles in many cellular processes, including the transmission of information. It is well established that the occurrence and development of gastric cancer, one of the four most common malignant tumors worldwide, involves the transmission of information. Based on the urgent need for the elucidation of the mechanism involved in this process, as well as advances in the diagnosis and treatment of gastric cancer, numerous reports have assessed the association between non-coding RNAs in exosomes and gastric cancer. The purpose of the present review was to summarize recent evidence on certain non-coding RNAs associated with the development, diagnosis and treatment of gastric cancer.
\end{abstract}

\section{Contents}

1. Introduction

2. MiRNA

3. LncRNA

4. CircRNA

5. Discussion

Correspondence to: Professor Hong-Wu Zhang, Department of Anatomy, Guangdong Provincial Key Laboratory of Construction and Detection in Tissue Engineering, Southern Medical University, 1023 Shatai South Road, Guangzhou, Guangdong 510515, P.R. China E-mail: zhanghwu@mail2.sysu.edu.cn

*Contributed equally

Key words: gastric cancer, exosomes, microRNAs, long non-coding RNAs, circular RNAs

\section{Introduction}

Gastric cancer is one of the most common types of tumors worldwide, with a high incidence reported in East Asia, Central Europe, Eastern Europe, and South America. According to the International Agency for Research on Cancer; approximately 951,000 individuals were diagnosed with gastric cancer and the disease caused approximately 723,000 deaths in 2012 (1). In China in 2015, approximately 679,000 and 498,000 patients were newly diagnosed with gastric cancer and expired due to the disease, respectively. According to these numbers, gastric cancer ranks second after lung cancer in terms of incidence and mortality (2). With the prevention and treatment of Helicobacter pylori infection and the improvement of diet, the rates of gastric cancer-related morbidity and mortality have exhibited a downward trend (3). Studies have shown that the 5 -year survival rate of patients with gastric cancer in most European countries and regions was only $30 \%$ (4). This disease seriously threatens the health of patients, severely affects their quality of life, and brings a heavy burden to society (5).

In 1987, Johnstone et al discovered vesicles secreted by sheep reticulocytes containing a variety of bioactive macromolecules during maturation, which were later termed exosomes (6). The main component of exosomes is lipid, which is rich in cholesterol, diglyceride, glycerophosphatide, phospholipid, and sphingomyelin or glycosylceramide (including sphingomyelin and ceramide) (7). In addition to these lipids, exosomes contain many types of bioactive lipids $(7,8)$. They also contain functional RNAs molecules, including messenger RNAs (mRNAs) and other non-coding RNAs, such as microRNAs (miRNAs), long non-coding RNAs (lncRNAs) $(9,10)$, and circular RNAs (circRNAs) (11). Many previous studies have demonstrated that exosomes exist in various fluids of the human body, including blood, amniotic fluid, urine, malignant ascites, cerebrospinal fluid, breast milk, saliva, lymph, and bile (12-14). Exosomes were initially thought to be unnecessary material discarded by cells (15). In subsequent studies, it was shown that exosomes, which used to be seen as molecular trash bins, carry molecules that can be absorbed and utilized by other cells $(16,17)$. Exosomes play an important role in normal physiological functions, such as lactation, inflammation, cell proliferation, immune response, and neurological function (18-20). In addition, they participate in thrombosis, diabetes, atherosclerosis, liver 
disease, neurodegenerative diseases (21-23), cancer (24), and other pathological processes.

In-depth studies have shown that gastric cancer is a complex structure composed of cancer cells and the stroma around them (25). Similar to normal cells, cancer cells require the transmission of information (26). However, it has been widely reported that RNAs (especially miRNAs in exosomes) closely participate in the transmission of information $(27,28)$. Other studies have shown that exosomes may also be associated with cancer cells, discarding their anti-cancer miRNAs and improving their tumorigenicity (29). In general, exosomes are closely involved in various changes in the tumor microenvironment, promoting the proliferation and migration of cancer cells (30).

Non-coding RNAs, which have been detected in exosomes, include miRNAs, IncRNAs, and circRNAs (9-11). They are related to many processes in tumor formation, including tumor growth, metastasis and drug resistance. This fact renders these non-coding RNAs potential targets for the diagnosis and treatment of cancer (31). However, the detailed mechanisms involved in these processes are unclear. For example, Zhang et al revealed the diagnostic value of exosomal lncRNA DLX6-AS1 in non-small cell lung cancer (32). Zhang et al found that the expression levels of miRNAs in exosomes are significantly different between patients with ovarian cancer and healthy individuals (33). Compared with the corresponding healthy state, the aforementioned differences are also present in clear cell renal carcinoma (34) and invasive breast cancer (35).

These are only a few examples of the value of non-coding RNAs in cancer. The following sections of this article will elaborate on the relationship between the non-coding RNAs in exosomes and gastric cancer.

\section{MiRNA}

Exosomal miRNA of gastric cancer. The discovery of miRNAs is one of the most important milestones in modern molecular biology. They were first discovered, identified, and named 'small temporal RNAs' by Lee et al (36) and Reinhart et al (37). miRNAs have a small molecular and mainly regulate the expression of mRNAs by binding to the 3'untranslated region (UTR) of the target gene. Their binding does not have one-to-one characteristics, i.e., a single UTR may have multiple miRNA binding sites, or a single miRNA can bind to multiple sites. This further indicates that miRNAs play a post-transcriptional regulatory role by mainly regulating mRNA stability and protein translation (38). The results suggest that these regulatory RNAs have complex post-transcriptional control mechanisms for gene expression. In addition, the expression of exosomal miRNAs varies between different cell and tissue types. As the mechanism of their function is slowly explored, it is possible for these molecules to be used as biomarkers for disease detection and targets for therapeutic interventions.

The role of exosomal miRNA in the diagnosis of gastric cancer. Although there are many biomarkers that can be used for the diagnosis of gastric cancer, they do not meet the demand for the early diagnosis of gastric cancer. New markers with better performance in the diagnosis of gastric cancer at an early stage are warranted. Since their detection in exosomes, several kinds of miRNAs have been found to be promising markers.

According to studies utilizing quantitative reverse transcriptase polymerase chain reaction, four kinds of miRNAs (miR-19b-3p, miR-17-5p, miR-30a-5p, and miR-106a-5p) have been related to the pathogenesis of gastric cancer. miR-19b and miR-106a are significantly overexpressed in patients with gastric cancer $(\mathrm{P}<0.0001)$. Receiver operating characteristic (ROC) analysis revealed that the area under curve (AUC) values for miR-106a-5p and miR19b-3p were 0.786 and 0.769 , respectively. Combined with ROC curve analysis, the highest AUC value in patients with gastric cancer and healthy controls was 0.814 . Further research showed that the characteristics of two miRNAs (miR-19b-3p and miR-106a-5p) correctly distinguished 19 of 20 gastric cancer serum samples $(95 \%$ sensitivity) and 18 of 20 healthy controls (90\% specificity). In addition, the above-mentioned miRNAs are associated with lymphatic metastasis of gastric cancer (stage I and II: $\mathrm{P}<0.01$; stage III and IV: $\mathrm{P}<0.05)$. Hence, miR-19b-3p and miR-106a-5p in serum exosomes are new potential biomarkers for the detection of gastric cancer (39).

In addition to the exosomal miRNAs, which have a detailed statistical proof, many other exosomal miRNAs have been found to be abnormally expressed in the exosomes of patients with gastric cancer. The expression of miR-217 (40), miR-27A (41), and miR-1290 (42) is pathologically high in the exosomes of patients with gastric cancer. miR-214, miR-221, and miR-222 are highly expressed in gastric cancer and their expression levels are closely related to lymph node metastasis, venous invasion, and tumor-node-metastasis (TNM) stage (43). In a study assessing the relationship between miR-130a and c-MYB mRNAs, Yang et al suggested that the levels of miR-130a in exosomes may be a potential biomarker for evaluating the invasion or metastasis of gastric cancer, although there is no more clinical statistical proof (44). Of note, increases in the expression of miRNAs are not the only observation in gastric cancer. The LET-7 family of miRNAs has an abnormal extracellular exosomal and intracellular content of AZ-P7a (29). The expression levels of miR-101 in gastric cancer tissues and gastric cancer cell lines are markedly lower that those recorded in normal gastric mucosa. Moreover, compared with healthy individuals, miR-101 in both exosomes and serum of patients with gastric cancer is significantly downregulated (45).

Above, we listed some miRNAs with significant differences in expression in exosomes. Some of those have been closely related to certain stages of gastric cancer. Relevant information regarding the miRNAs mentioned in this article is presented in Table I. Although some exhibit abnormal expression levels, there was no statistical proof. Moreover, these miRNAs have not been compared with the commonly used diagnostic markers of gastric cancer. Perhaps in the near future, these miRNAs can help to more accurately diagnose gastric cancer, determine the stage of disease, and guide the clinical treatment.

Promoting mechanism and role of exosomal miRNA in the treatment of gastric cancer. It is established that the development of gastric cancer is not the result of a single factor, such as cell mutation, growth, malignant maintenance, anti-apoptosis, vascular growth and cell metastasis. These 
Table I. miRNAs in exosomes of patients with gastric cancer.

\begin{tabular}{clllcc}
\hline Accession no. & Gene ID & Name & $\begin{array}{c}\text { Source of } \\
\text { exosomes }\end{array}$ & Expression & $\begin{array}{c}\text { Related } \\
\text { target/molecule }\end{array}$ \\
\hline- & - & LET-7 family & GC cell & - & RAS, HMGA2 \\
(Refs.) & (29) \\
NC_000013.11 & 406980 & miR-19b & Serum & UP & Unknown \\
NC_000023.11 & 406899 & miR-106a & Serum & UP & Inknown \\
NC_000002.12 & 406999 & miR-217 & GC cell & UP & CDH1 \\
NC_000019.10 & 407018 & miR-27a & GC cell & UP & CSRP2 \\
NC_000001.11 & 100302276 & miR-1290 & Serum & UP & NKD1 \\
NC_000001.11 & 406996 & miR-214 & GC cell & UP & Unknown \\
NC_000023.11 & 407006 & miR-221 & GC cell & UP & Unknown \\
NC_000023.11 & 407007 & miR-222 & GC cell & UP & Unknown \\
NC_000011.10 & 406919 & miR-130a & GC cell & UP & c-MYB \\
NC_000007.14 & 407014 & miR-101 & GC cell & DOWN & MCL1, ZEB1 \\
NC_000011.10 & 406992 & miR-25 & EAC cell & UP & PTEN, AIFM3 \\
\end{tabular}

In the article, let-7 family and miR-101 refers to a group. Certain information is not listed in detail. If necessary, please refer to the original articles. GC, gastric cancer; EAC, esophageal adenocarcinoma; RAS, rat sarcoma virus, here refers to oncogenes firstly discovered from rat sarcoma virus; HMGA2, high mobility group AT-hook 2; CDH1, cadherin 1; CSRP2, cysteine and glycine rich protein 2; NKD1, NKD inhibitor of WNT signaling pathway 1; c-MYB, here refers to MYB proto-oncogene, transcription factor; MCL1, myeloid cell leukemia 1; ZEB1, zinc finger E-box binding homeobox 1; PTEN, gene of phosphate and tension homology deleted on chromsome ten; AIFM3, apoptosis inducing factor mitochondria associated 3; UP, upregulated; Down, downregulated; miR, microRNA.

factors play an important role in the occurrence and development of gastric cancer. With the gradual discovery of exosomal miRNAs, scientists have found that some affect the formation and development of gastric cancer in many stages, through complex pathways.

Firstly, regarding the growth stage of gastric cancer, there is a negative correlation between the expression of miR-217 and cadherin 1 (CDH1; also known as E-cadherin). The former contributes substantially to intercellular adhesions as a transmembrane glycoprotein. It has also been proved to be a tumor suppressor and its expression is decreased in gastric cancer. Studies utilizing double luciferase analysis and immunoblotting showed that miR-217 targets CDH1. Overexpression of miR-217 can enhance the proliferation of gastric cancer cells. This leads to the decrease of exosomal CDH1, and this effect is also observed in the microenvironment. These findings reveal part of the function of miR-217 (40) and an important part of the growth of gastric cancer.

The microenvironment is particularly important in the growth of malignant tumors (46). As part of the tumor microenvironment, cancer-related fibroblasts exert a negative effect on patients (47). High miR-27A expression in exosomes can induce the reprogramming of fibroblasts into cancer-related fibroblasts and promote the proliferation, migration, and metastasis of malignant cells. Overexpression of miR-27A cancer-related fibroblasts significantly increased the malignant degree of gastric cancer cells. Further investigation revealed that cysteine and glycine-rich protein 2 (CSRP2) is the downstream target gene of miR-27A, and its downregulation increases the replication of gastric cancer cells (41).

Angiogenesis is involved in almost the entire course of cancer, including occurrence $(48,49)$, progression $(50)$, invasion, and metastasis $(51,52)$. Studies have shown that c-MYB is a transcription factor affecting the growth of blood vessels (53). The expression of miR-130a is significantly increased in gastric cancer cells and their exosomes. Bioinformatics analysis combined with luciferase analysis showed that miR-130a directly targeted 30 UTR of c-MYB mRNA. Subsequently, they also confirmed that the overexpression of miR-130a significantly promotes the growth and angiogenesis of implanted tumors in mice (44).

Anti-apoptosis is important for cancer cells. MCL1, which belongs to the BCL2 family, is often highly expressed in cancer cells and involved in anti-apoptosis (54). Zinc finger E-box binding homeobox 1 (ZEB1) can boost the invasiveness of epithelial tumors. It exerts its effects by inhibiting the E-cadherin promoter and inducing epithelial-stromal transformation. Studies have shown that restored levels of miR-101, which exists in malignant cells and exosomes, can induce apoptosis by inhibiting MCL1; moreover, they inhibit cell migration and invasion by regulating ZEB1. Further research suggested that the decrease of tumor suppressor miRNA-101 is closely related to tumor progression (45). Exosomes from esophageal adenocarcinoma can also affect the gastrointestinal tract, assisting gastric cancer cells to fight apoptosis and create a favorable environment. The gastrointestinal cells, which treated with esophageal adenocarcinoma-derived extracellular vesicles (C-EV), are more crowded, compact, and multilayered and contained fewer lumens than control group. Further studies showed that, in the control group treated with $\mathrm{C}-\mathrm{EV}$, the expression levels of miR-25 and miR-210 were significantly higher, whereas those of phosphatase and tensin homolog (PTEN) and apoptosis-inducing factor mitochondria associated 3 (AIFM3) were significantly decreased. PTEN 


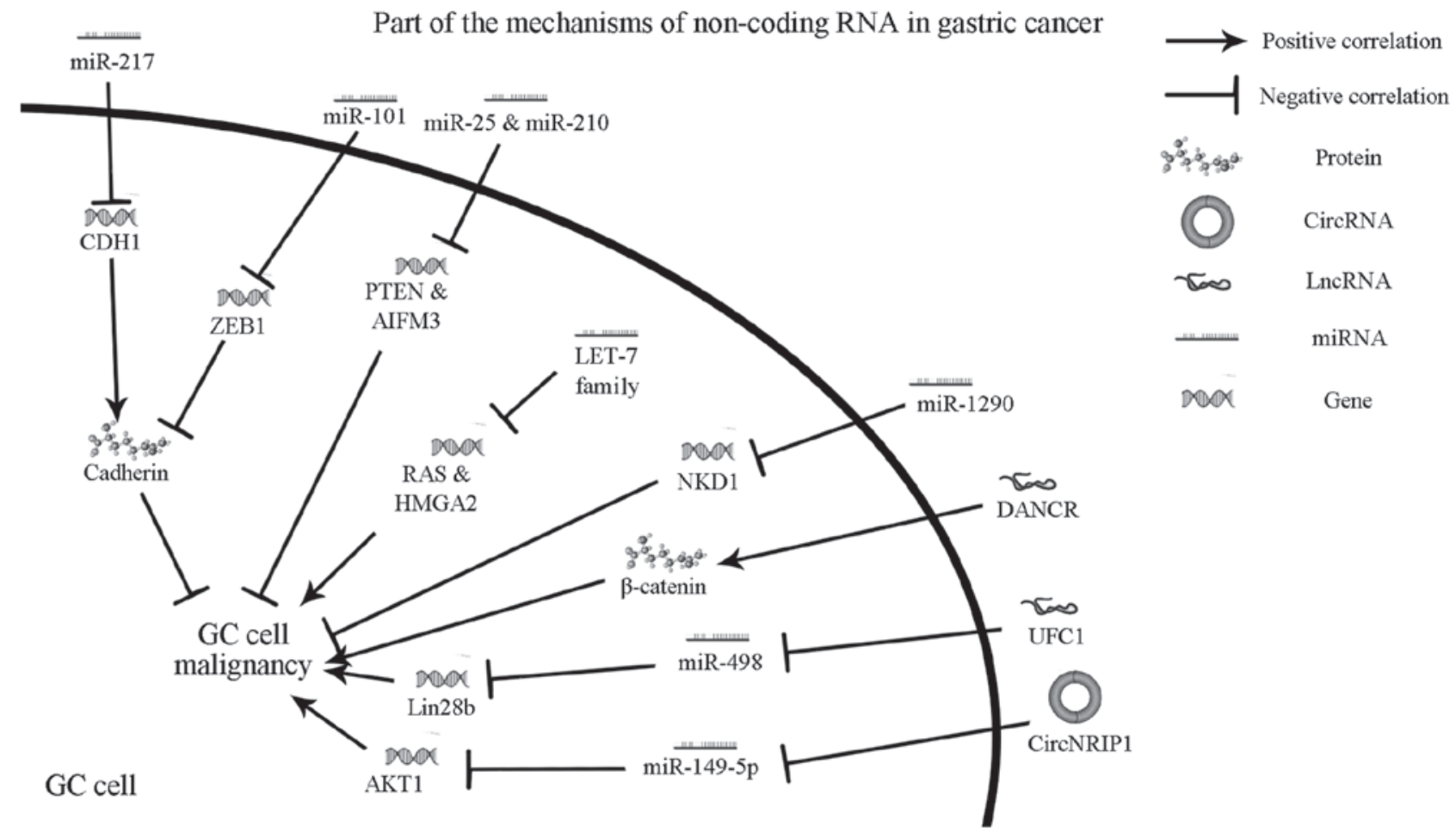

Figure 1. Mechanisms of non-coding RNA in GC. Various mechanisms of non-coding RNA act on GC cells via exosomes. Related molecules are also described. miRNA, microRNA; GC, gastric cancer; CDH1, cadherin 1; ZEB1, zinc finger E-box binding homeobox 1; PTEN, phosphatase and tensin homolog; AIFM3, apoptosis-inducing factor mitochondria associated 3; RAS, rat sarcoma virus, here refers to oncogenes firstly discovered from rat sarcoma virus; HMGA2, high-mobility group AT-hook 2; NKD1, NKD inhibitor of WNT signaling pathway 1; DANCR, anti-differentiation antagonizing non-protein coding RNA; UFC1, ubiquitin-fold modifier conjugating enzyme 1; lncRNA, long non-coding RNA; circRNA, circular RNA.

and AIFM3 are apoptotic genes. When the expression is low, abnormal proliferative cells may escape the fate of apoptosis. However, the effects of C-EV on co-cultured gastrointestinal tract could be reversed by inhibiting the high expression levels of miR-25 and miR-210 (55).

The development of gastric cancer is promoted through the secretion of miRNAs by exosomes that are conducive to the growth of cancer cells. In addition, gastric cancer cells can also discard miRNAs that impede their growth through the exosomes. The LET-7 family of miRNAs plays a major role as tumor suppressor genes (56), targeting RAS and high-mobility group AT-hook 2 (HMGA2) (57). A study showed that the LET-7 miRNAs were abnormally expressed in exosomes of AZ-P7a cells. Further research indicated that AZ-P7a cells maintain their tumorigenicity and metastatic tendency by selectively secreting LET-7 miRNAs into exosomes entering the extracellular environment (29).

Previous studies have interfered with the abnormal expression of miRNAs in exosomes, which may play a role in the development of gastric cancer cells, hindering or reversing the growth of gastric cancer cells. The expression of miR-214, miR-221 and miR-222 in exosomes of patients with gastric cancer exhibits high levels. A study showed that exosomes, which are secreted by gastric cancer-derived mesenchymal stem cells, deliver miR-221 to HGC-27 cells and promote malignancy. Inhibition of miR-221 can block the tumor support provided by gastric cancer-derived mesenchymal stem cells (43). In addition, the high expression of miR-1290 in exosomes of patients with gastric cancer has been confirmed. Subsequently, fluorescence results showed that NKD inhibitor of WNT signaling pathway 1
(NKD1) mRNA is the direct target of miR-1290. In contrast, overexpression of NKD1 could attenuate the effect of miR-1290 on gastric cancer cells. In summary, exosomal miR-1290 can enhance the malignancy of gastric cancer cells by targeting NKD1 mRNA, to downregulate its expression (42).

According to this evidence, exosomal miRNAs play an important role in the formation and development of gastric cancer. Moreover, there is a variety of mechanisms and molecular pathways involved in this process. For example, these miRNAs can directly assist cancer cells to grow, invade, and resist apoptosis. Moreover, they affect the nearby microenvironment and discard miRNAs that prevent cancer cell growth through exosomes. Relevant information regarding the miRNAs mentioned in this article is presented in Table I and Fig. 1. We hypothesized that such diverse and complex mechanisms suggest the involvement of many alternative pathways hindering the formation and development of gastric cancer. For example, this idea has been confirmed in experiments targeting miR-221 (43) and miR-1290 (42). We believe that the aforementioned mechanisms and molecular pathways can be used as targets to hinder the formation and development of gastric cancer cells, although there is no conclusive research evidence. This may be the goal of future research. Although these findings are preliminary, they can be used as routes and guidance for follow-up research.

\section{LncRNA}

Exosomal lncRNA of gastric cancer. LncRNAs are $>200$ base pairs long (i.e., longer than miRNAs). Currently, there is limited research on lncRNAs. Although $>20,000$ lncRNAs have already 
been annotated, our knowledge of lncRNAs remains limited compared with that on miRNAs. It is established that they play a role mainly by regulating the transcription of genes that encode proteins (58). Notably, scientists have also found some traces of lncRNAs in the exosomes of patients with gastric cancer.

The role of exosomal lncRNA in the diagnosis of gastric cancer. Some lncRNAs have shown excellent potential as diagnostic markers of gastric cancer. Through the combinatorial analysis of RNA sequencing results, lncUEGC1 and lncUEGC2 in exosomes were shown to be highly expressed in patients with gastric cancer. The same results were obtained in gastric cancer cell culture medium. Of course, IncUEGC1 and 1ncUEGC2 are also present in plasma. Studies have shown that almost all plasma lncUEGC1 is wrapped in exosomes, and exosomes can protect them from degradation by ribonuclease. The diagnostic accuracy of exosomal lncUEGC1 has been evaluated. It exhibited AUC values of 0.8760 and 0.8406 in discriminating patients with early gastric cancer from healthy individuals and those with premalignant chronic atrophic gastritis, respectively. Notably, this diagnostic accuracy is higher than that of carcinoembryonic antigen (59). In addition, the expression levels of anti-differentiation antagonizing non-protein coding RNA (DANCR) targeting lncRNA-LET in serum exosomes of patients with gastric cancer are high. The results of a ROC curve analysis yielded a DANCR AUC value of 0.777. As indicator, DANCR can indirectly reflect the levels of lncRNA-LET and diagnose gastric cancer, and performs better than traditional serological markers (carcinoembryonic antigen and carbohydrate antigen 19-9 [CA19-9]). Based on this evidence, it was suggested that DANCR may be a biomarker for the diagnosis of gastric cancer (60). In addition, Hao et al suggested that DANCR can be used as a prognostic index for the growth and tumorigenicity of gastric cancer cells (61). Furthermore, through analysis of lncRNA HOTTIP in the serum exosomes of 246 subjects, a study showed that lncRNA HOXA distal transcript antisense RNA (HOTTIP) has higher expression levels in exosomes of patients with gastric cancer than in those of healthy individuals. The expression levels were significantly related to the depth of invasion and TNM stage. Moreover, the AUC of HOTTIP in exosomes was 0.827 , indicating that its diagnostic ability is higher than that of carcinoembryonic antigen, CA19-9, and CA72-4. The Kaplan-Meier analysis showed a correlation between increased levels of exosomal HOTTIP and poor overall survival (log-rank $\mathrm{P}<0.001$ ). Univariate and multivariate COX (proportional hazards model) analyses revealed that overexpression of exosomal HOTTIP is an independent prognostic factor in patients with gastric cancer $(\mathrm{P}=0.027)$. These results suggested that HOTTIP in exosomes may be a potential biomarker in the diagnosis and prognosis of gastric cancer (62).

In conclusion, these IncRNAs demonstrated excellent diagnostic performance in the laboratory. Although further research is warranted to assess their utility in the clinical setting, these lncRNAs show promise for the diagnosis of gastric cancer.

Promoting mechanism and role of exosomal IncRNA in the treatment of gastric cancer. DANCR was first detected at high levels in liver cancer (63). In subsequent studies, DANCR was also proved to be expressed at high levels in exosomes of patients with gastric cancer (60). The expression of DANCR is closely related to tumor size, TNM stage, lymph node metastasis, and depth of invasion. Knockout of the DANCR gene can also inhibit epithelial-mesenchymal transition, as well as the migration and invasion of gastric cancer cells. Spalt-like transcription factor 4 (SALL4) activates DANCR. Moreover, the $\beta$-catenin pathway is activated as a result of the overexpression of DANCR (64). Further studies have shown that IncRNA-LET is the target gene of DANCR. DANCR binds to enhancer of zeste 2 polycomb repressive complex 2 subunit (EZH2) and histone deacetylase 3 (HDAC3) to silence lncRNA-LET, and subsequently regulate the migration and invasion of gastric cancer. In summary, the DANCR-lncRNA-LET mechanism plays an important role in the migration and invasion of gastric cancer cells, revealing the new epigenetic mechanism of lncRNA-LET silencing (65).

A study revealed that the prognosis of gastric cancer patients with elevated levels of exosomal UFC1 is poor. Knockout of the UFC1 gene successfully hindered the proliferation, migration, and invasion of gastric cancer cells, while its overexpression promoted these processes. Although the mechanism are not fully understood, it is clear that UFC1 can act on miR-498 and downregulate the expression of LIN28B (66).

LncRNAs may be more complex than miRNAs in terms of the mechanism. The impact of changes of lncRNAs may be achieved by interfering with miRNAs, as well as other effects. The complex mechanisms of lncRNAs need to be further investigated. Relevant information regarding the lncRNAs mentioned in this article is presented in Fig. 1. The abnormal expression of lncRNAs in exosomes of patients with gastric cancer and the possible mechanism provide a new idea for treatment. For example, as mentioned above, regulation of UFC1 has a certain effect on the growth of gastric cancer cells (66), although more in-depth and detailed studies are needed to extrapolate these laboratory data to clinical practice.

\section{CircRNA}

Exosomal circRNA of gastric cancer. Covalently closed circRNAs were originally found in plant viruses (67). Unlike lncRNAs and miRNAs, circRNAs do not have a $5^{\prime}$ head and $3^{\prime}$ tail, forming a ring in a covalently closed manner. They were previously considered by-products of indirect errors and thus, their role in life was ignored $(68,69)$. High expression of circRNAs was first found in the brains of humans and mice. Studies have shown that it functions as a miR-7 sponge. The circular transcript was later termed circRNA sponge for miR-7 (CIRS-7). More than 70 selectively conserved miRNA targets are present in CIRS-7. It exerts its effect by binding to the Argonaute protein in a miR-7-dependent manner. Circular CIRS-7 may act as a miRNA sponge binding to miR-7, downregulating the expression of miR-7 and regulating downstream oncogenes to promote tumor cell proliferation and metastasis. Moreover, research revealed that the testis-specific circRNA sex-determining region $\mathrm{Y}$ (Sry) serves as a miR138 sponge. This finding suggested that the miRNA sponge effect of circRNAs is not unique to some kinds of circRNAs. This study is the first functional analysis of naturally expressed circRNAs (70). Although the evidence related to exosomal circRNAs in terms of specificity, conservatism, and stability 
is not as rich as that for miRNAs and lncRNAs, the confirmed existence of circRNAs in exosomes provides a new direction for the diagnosis and targeted therapy of tumors.

The role of exosomal circRNA in the diagnosis of gastric cancer. Analysis of circRNA hsa_circ_0065149 in exosomes collected from individuals with gastric cancer reported differences in the expression levels between four stages: Healthy stomach, gastritis, intestinal metaplasia, and gastric cancer. Hsa_circ_0065149 in exosomes, as a molecular tool for the screening of early gastric field cancerization, has higher sensitivity and specificity than traditional clinical biomarkers. Statistical analysis showed that the survival time of gastric cancer patients with low levels of hsa_circ_0065149 is longer $(\mathrm{P}<0.020)$. Moreover, the levels of hsa_circ_0065149 in patients is significantly correlated to tumor diameter and nerve invasion (71).

In addition to the circRNAs that are abnormally expressed in the exosomes of patients with gastric cancer, an increasing number of circRNAs associated with gastric cancer are being discovered, such as circFNDC3B (72) and hsa_circ_0000144 (73), and hsa_circ_0005654 (74). However, thus far, there is not enough evidence to prove that they are present in the exosomes and helpful in the diagnosis.

Promoting mechanism and role of exosomal circRNA in the treatment of gastric cancer. The supply of energy is of critical importance for the growth and proliferation of cancer cells. Some kinds of cancer cells can alter several points of the phosphatidy linositide 3-kinases/protein kinase $\mathrm{B}$ (PI3K/AKT) signaling pathway to change their metabolism, in order to gain more selective advantages than other cells (75). The AKT/mechanistic target of rapamycin kinase (AKT/MTOR) axis is a classical signaling pathway, which can meet the needs of gastric cancer cell proliferation through the Warburg effect (76). In addition, AKT/MTOR can also promote anabolism (e.g., protein synthesis) and prevent catabolic activity (e.g., autophagy), and the final effect is beneficial to the growth of gastric cancer cells (77). Further study showed that the AKT/MTOR axis plays a positive role in epithelial-mesenchymal transition and is closely related to tumor metastasis $(78,79)$. A recent study showed that exosomal circRNA circNRIP1 can sponge miR-149-5p and alter the expression of AKT1. Also, inhibition of exosomal circNRIP1 can affect the AKT1/MTOR signaling pathway and block the malignant behavior of gastric cancer cells (80).

Research on circRNAs is at a preliminary stage compared with that for miRNAs and lncRNAs. Relevant information regarding the circRNAs mentioned in this article is presented in Fig. 1. However, it is expected that more mechanisms of circRNAs will be revealed in the future. CircRNAs can also be involved in the occurrence and development of gastric cancer. Therefore, it may be possible to treat gastric cancer by targeting circRNAs. More in-depth research will provide new directions for the treatment of gastric cancer.

\section{Discussion}

Gastric cancer poses a threat to human health and is responsible for a substantial number of death worldwide. Patients with gastric cancer are often diagnosed at a late stage of the disease, missing the optimal period for treatment $(2,81)$. Therefore, investigation of the pathogenesis and discovery of effective diagnostic and therapeutic approaches is of great importance.

The exosomes are stable in vitro and can be stored at $4^{\circ} \mathrm{C}$ for $96 \mathrm{~h}$ or at $-70^{\circ} \mathrm{C}$ for longer periods of time (12). In addition to serum, exosomes are also found in various fluids of the human body, including blood, amniotic fluid, urine, malignant ascites, cerebrospinal fluid, breast milk, saliva, lymph, and bile (12-14). Moreover, the number of RNAs in the exosomes of gastric cancer was several-fold higher than that of normal gastric mucosal epithelial cells (82). These excellent properties render exosomes and exosomal non-coding RNAs ideal biomarker candidates. An increasing number of studies on exosomal non-coding RNAs obtained from patients with gastric cancer found that some important indexes of non-coding RNAs are better than those of traditional gastric cancer markers (e.g., lncRNA DANCR) (60).

In addition to diagnosis, elucidation of the mechanism of exosomal non-coding RNAs involved in promoting or inhibiting the development of gastric cancer may influence the therapeutic strategy. Intervention with exosomal non-coding RNAs is expected to become a new direction for the treatment of gastric cancer.

It is thought that effective methods for the diagnosis and treatment of gastric cancer through the use of exosomal non-coding RNAs will be developed in the near future. However, further research is warranted to translate these findings from the laboratory to clinical practice.

\section{Acknowledgements}

Not applicable.

\section{Funding}

The present study was supported by the Natural Science Foundation of Guangdong Province in China (grant no. 2016A030313602), Guangdong Provincial Science and Technology Plan Projects (grant no. 2016B090913004) and the Science and Technology Program of Guangzhou, China (grant no. 201604020007).

\section{Availability of data and materials}

Not applicable.

\section{Authors' contributions}

PG was responsible for writing and revising the manuscript. $\mathrm{DH}$ was responsible for the collection and analysis of relevant literature and contributed to the revision of the manuscript. HWZ designed the project and acquired funding and resources. JW and WL designed and drew of the table and figure, and participated in the revision of the manuscript. All authors read and approved the final manuscript.

\section{Ethics approval and consent to participate}

Not applicable. 


\section{Patient consent for publication}

Not applicable.

\section{Competing interests}

The authors declare that they have no competing interests.

\section{References}

1. Torre LA, Bray F, Siegel RL, Ferlay J, Lortet-Tieulent J and Jemal A: Global cancer statistics, 2012. CA Cancer J Clin 65: 87-108, 2015

2. Chen W, Zheng R, Baade PD, Zhang S, Zeng H, Bray F, Jemal A, Yu Qx and He J: Cancer statistics in China, 2015. CA Cancer J Clin 66: 115-132, 2016.

3. Choi IJ, Kook MC, Kim YI, Cho JS, Lee JY, Kim CG, Park B and Nam BH: Helicobacter pylori therapy for the prevention of metachronous gastric cancer. New Engl J Med 378: 1085-1095, 2018.

4. Sitarz R, Skierucha M, Mielko J, Offerhaus GJA, Maciejewski R and Polkowski WP: Gastric cancer: Epidemiology, prevention, classification, and treatment. Cancer Manag Res 10: 239-248, 2018.

5. Zhou M, Wang H, Zhu J, Chen W, Wang L, Liu S, Li Y, Wang L, Liu Y, Yin P, et al: Cause-specific mortality for 240 causes in China during 1990-2013: A systematic subnational analysis for the Global Burden of disease study 2013. Lancet 387: 251-272, 2016.

6. Johnstone RM, Adam M, Hammond JR, Orr L and Turbide C: Vesicle formation during reticulocyte maturation. Association of plasma membrane activities with released vesicles (exosomes). J Biol Chem 262: 9412-9420, 1987.

7. Record M, Carayon K, Poirot M and Silvente-Poirot S: Exosomes as new vesicular lipid transporters involved in cell-cell communication and various pathophysiologies. Biochim Biophys Acta 1841: 108-120, 2014

8. Subra C, Grand D, Laulagnier K, Stella A, Lambeau G, Paillasse M, Medina PD, Monsarrat B, Perret B, Poirot SS, et al: Exosomes account for vesicle-mediated transcellular transport of activatable phospholipases and prostaglandins. J Lipid Res 51 2105-2120, 2010.

9. Valadi H, Ekström K, Bossios A, Sjöstrand M, Lee JJ and Lötvall JO: Exosome-mediated transfer of mRNAs and microRNAs is a novel mechanism of genetic exchange between cells. Nat Cell Biol 9: 654-659, 2007.

10. Gezer U, Özgur E, Cetinkaya M, Isin M and Dalay N: Long non-coding RNAs with low expression levels in cells are enriched in secreted exosomes. Cell Biol Int 38: 1076-1079, 2014.

11. Fanale D, Taverna S, Russo A and Bazan V: Circular RNA in exosomes. Adv Exp Med Biol 1087: 109-117, 2018.

12. Taylor DD and Gercel-Taylor C: MicroRNA signatures of tumor-derived exosomes as diagnostic biomarkers of ovarian cancer. Gynecol Oncol 110: 13-21, 2008.

13. Simpson RJ, Lim JWE, Moritz RL and Mathivanan S: Exosomes: Proteomic insights and diagnostic potential. Expert Rev Proteomics 6: 267-283, 2009.

14. Gallo A, Tandon M, Alevizos I and Illei GG: The majority of MicroRNAs detectable in serum and saliva is concentrated in exosomes. PLoS One 7: e30679, 2012.

15. Johnstone RM: The Jeanne Manery-fisher memorial lecture 1991. Maturation of reticulocytes: Formation of exosomes as a mechanism for shedding membrane proteins. Biochem Cell Biol 70: 179-190, 1992.

16. Bang $C$ and Thum T: Exosomes: New players in cell-cell communication. Int J Biochem Cell Biol 44: 2060-2064, 2012.

17. Tang MK and Wong AS: Exosomes: Emerging biomarkers and targets for ovarian cancer. Cancer Lett 367: 26-33, 2015.

18. Simhadri VR, Reiners KS, Hansen HP, Topolar D, Simhadri VL, Nohroudi K, Kufer TA, Engert A and von Strandmann EP: Dendritic cells release HLA-B-associated Transcript-3 positive exosomes to regulate natural Killer function. PLoS One 3: e3377, 2008.

19. Admyre C, Johansson SM, Qazi KR, Filén JJ, Lahesmaa R, Norman M, Neve EPA, Scheynius A and Gabrielsson S: Exosomes with immune modulatory features are present in human breast milk. J Immunol 179: 1969-1978, 2007.
20. Gu Y,Li M, Wang T, Liang Y,Zhong Z, Wang X, Zhou Q, Chen L, Lang Q, He Z, et al: Lactation-related MicroRNA expression profiles of porcine breast milk exosomes. PLoS One 7: e43691, 2012.

21. Pontes Azevedo LC, Janiszewski M, Pontieri V, Pedro MDA, Bassi E, Tucci PJF and Laurindo FRM: Platelet-derived exosomes from septic shock patients induce myocardial dysfunction. Crit Care 11: R120, 2007.

22. Masyuk AI, Masyuk TV and LaRusso NF: Exosomes in the pathogenesis, diagnostics and therapeutics of liver diseases. J Hepatol 59: 621-625, 2013.

23. Vella LJ, Sharples RA, Nisbet RM, Cappai R and Hill AF: The role of exosomes in the processing of proteins associated with neurodegenerative diseases. Eur Biophys J 37: 323-332, 2008.

24. Rak J and Guha A: Extracellular vesicles-vehicles that spread cancer genes. Bioessays 34: 489-497, 2012.

25. Sund M and Kalluri R: Tumor stroma derived biomarkers in cancer. Cancer Metastasis Rev 28: 177-183, 2009.

26. Kalluri R and Zeisberg M: Fibroblasts in cancer. Nat Rev Cancer 6: 392-401, 2006.

27. Skog J, Wuerdinger T, van Rijn S, Meijer DH, Gainche L, Esteves MS, Curry WT Jr, Carter BS, Krichevsky AM and Breakefield XO: Glioblastoma microvesicles transport RNA and proteins that promote tumour growth and provide diagnostic biomarkers. Nat Cell Biol 10: 1470-1476, 2008.

28. Kogure T, Lin WL, Yan IK, Braconi C and Patel T: Intercellular Nanovesicle-mediated microRNA transfer: A mechanism of environmental modulation of hepatocellular cancer cell growth. Hepatology 54: 1237-1248, 2011.

29. Ohshima K, Inoue K, Fujiwara A, Hatakeyama K, Kanto K, Watanabe Y, Muramatsu K, Fukuda Y, Ogura SI, Yamaguchi K and Mochizuki T: Let-7 MicroRNA family is selectively secreted into the extracellular environment via exosomes in a metastatic gastric cancer cell line. PLoS One 5: e13247, 2010.

30. Pan L, Liang W, Fu M, Huang ZH, Li X, Zhang W, Zhang P, Qian H, Jiang PC, Xu WR and Zhang X: Exosomes-mediated transfer of long noncoding RNA ZFAS1 promotes gastric cancer progression. J Cancer Res Clini Oncol 143: 991-1004, 2017.

31. Melo SA, Sugimoto H, O'Connell JT, Kato N, Villanueva A, Vidal A, Qiu L, Vitkin E, Perelman LT, Melo CA, et al: Cancer exosomes perform cell-independent microRNA biogenesis and promote tumorigenesis. Cancer Cell 26: 707-721, 2014.

32. Zhang X, Guo H, Bao Y, Yu H, Xie D and Wang X: Exosomal long non-coding RNA DLX6-AS1 as a potential diagnostic biomarker for non-small cell lung cancer. Oncol Lett 18 : 5197-5204, 2019

33. Zhang H, Xu S and Liu X: MicroRNA profiling of plasma exosomes from patients with ovarian cancer using high-throughput sequencing. Oncol Lett 17: 5601-5607, 2019.

34. Crentsil VC, Liu H and Sellitti DF: Comparison of exosomal microRNAs secreted by 786-O clear cell renal carcinoma cells and HK-2 proximal tubule-derived cells in culture identifies microRNA-205 as a potential biomarker of clear cell renal carcinoma. Oncol Lett 16: 1285-1290, 2018.

35. Yoshikawa M,Iinuma H,Umemoto Y, Yanagisawa T,Matsumoto A and Jinno H: Exosome-encapsulated microRNA-223-3p as a minimally invasive biomarker for the early detection of invasive breast cancer. Oncol Lett 15: 9584-9592, 2018.

36. Lee RC, Feinbaum RL and Ambros V: The C. elegans heterochronic gene lin-4 encodes small RNAs with antisense complementarity to lin-14. Cell 75: 843-854, 1993.

37. Reinhart BJ, Slack FJ, Basson M, Pasquinelli AE, Bettinger JC, Rougvie AE, Horvitz HR and Ruvkun G: The 21-nucleotide let-7 RNA regulates developmental timing in Caenorhabditis elegans. Nature 403: 901-906, 2000.

38. Shukla GC, Singh J and Barik S: MicroRNAs: Processing, maturation, target recognition and regulatory functions. Mol Cell Pharmacol 3: 83-92, 2011.

39. Wang N, Wang L, Yang Y, Gong L, Xiao B and Liu X: A serum exosomal microRNA panel as a potential biomarker test for gastric cancer. Biochem Biophys Res Commun 493: 1322-1328, 2017.

40. Li W and Gao YQ: MiR-217 is involved in the carcinogenesis of gastric cancer by down-regulating $\mathrm{CDH} 1$ expression. Kaohsiung J Med Sci 34: 377-384, 2018.

41. Wang J, Guan X, Zhang Y, Ge S, Zhang L, Li H, Wang X, Liu R, Ning T, Deng T, et al: Exosomal miR-27a derived from gastric cancer cells regulates the transformation of fibroblasts into cancer-associated fibroblasts. Cell Physiol Biochem 49: 869-883, 2018 . 
42. Jiying H, Manru S, Meizhu Y, Ying C, Zhenjun G and Xin M Exosome-mediated transfer of miR-1290 promotes cell proliferation and invasion in gastric cancer via NKD1. Acta Biochim Biophys Sin (Shanghai) 51: 900-907, 2019.

43. Wang M, Zhao C, Shi H, Zhang B, Zhang L, Zhang X, Wang S, Wu X, Yang T, Huang F, et al: Deregulated microRNAs in gastric cancer tissue-derived mesenchymal stem cells: Novel biomarkers and a mechanism for gastric cancer. Br J Cancer 110: 1199-1210, 2014.

44. Yang H, Zhang H, Ge S, Ning T, Bai M, Li J, Li S, Sun W, Deng T, Zhang L, et al: Exosome-derived miR-130a activates angiogenesis in gastric cancer by targeting C-MYB in vascular endothelial cells. Mol Ther 26: 2466-2475, 2018.

45. Imamura T, Komatsu S, Ichikawa D, Miyamae M, Okajima W, Ohashi T, Kiuchi J, Nishibeppu K, Kosuga T, Konishi H, et al: Low plasma levels of miR-101 are associated with tumor progression in gastric cancer. Oncotarget 8: 106538-106550, 2017.

46. Kessenbrock K, Plaks V and Werb Z: Matrix Metalloproteinases: Regulators of the tumor microenvironment. Cell 141: 52-67, 2010.

47. Hwang RF, Moore T, Arumugam T, Ramachandran V, Amos KD, Rivera A, Ji B, Evans DB and Logsdon CD: Cancer-associated stroma fibroblasts promote pancreatic tumor progression. Cancer Res 68: 918-926, 2008.

48. Albini A, Tosetti F, Li VW, Noonan DM and Li WW: Cancer prevention by targeting angiogenesis. Nat Rev Clin Oncol 9: 498-509, 2012

49. Bergers $G$ and Benjamin LE: Tumorigenesis and the angiogenic switch. Nat Rev Cancer 3: 401-410, 2003.

50. Talasila KM, Soentgerath A, Euskirchen P, Rosland GV, Wang J, Huszthy PC, Prestegarden L, Skaftnesmo KO, Sakariassen PQ, Eskilsson E, et al: EGFR wild-type amplification and activation promote invasion and development of glioblastoma independent of angiogenesis. Acta Neuropathol 125: 683-698, 2013.

51. Cully M: Tumour vessel normalization takes centre stage. Nat Rev Drug Discovery 16: 87-87, 2017.

52. Viallard C and Larrivee B: Tumor angiogenesis and vascular normalization: Alternative therapeutic targets. Angiogenesis 20: 409-426, 2017.

53. Ramsay RG, Barton AL and Gonda TJ: Targeting c-Myb expression in human disease. Expert Opin Ther Targets 7: 235-248, 2003

54. Wang L, Liu Q, Zhou W, Shao H, Li F and Li X: Prognostic role of myeloid cell Leukemia-1 protein (Mcl-1) expression in human gastric cancer. J Surg Oncol 100: 396-400, 2009.

55. Ke X, Yan R, Sun Z, Cheng Y, Meltzer A, Lu N, Shu X, Wang Z, Huang B, Liu X, et al: Esophageal adenocarcinoma-derived extracellular vesicle MicroRNAs induce a neoplastic phenotype in gastric organoids. Neoplasia 19: 941-949, 2017.

56. Roush S and Slack FJ: The let-7 family of microRNAs. Trends Cell Biol 18: 505-516, 2008.

57. Boyerinas B, Park SM, Hau A, Murmann AE and Peter ME: The role of let-7 in cell differentiation and cancer. Endocrine Related Cancer 17: F19-F36, 2010.

58. Kolat D, Hammouz R, Bednarek AK and Pluciennik E: Exosomes as carriers transporting long noncoding RNAs: Molecular characteristics and their function in cancer (Review). Mol Med Rep 20: 851-862, 2019.

59. Lin LY, Yang L, Zeng Q, Wang L, Chen ML, Zhao ZH, Ye GD, Luo QC, Lv PY, Guo QW, et al: Tumor-originated exosomal lncUEGC1 as a circulating biomarker for early-stage gastric cancer. Mol Cancer 17: 84, 2018.

60. Yang L, Lei P, Wei L, Peng Z, Hui Q, Wenrong X, Zhijian Z, Pengcheng $J$ and $\mathrm{Xu} \mathrm{Z}$ : Detection and clinical value of serum exosomal DANCR in gastric cancer patients. Chin J Clin Lab Sci 35: 171-174, 2017. (In Chinese)

61. Hao YP, Qiu JH, Zhang DB and Yu CG: Long non-coding RNA DANCR, a prognostic indicator, promotes cell growth and tumorigenicity in gastric cancer. Tumour Biol 39: 1010428317699798,2017

62. Zhao R, Zhang Y, Zhang X, Yang Y, Zheng X, Li X, Liu Y and Zhang Y: Exosomal long noncoding RNA HOTTIP as potential novel diagnostic and prognostic biomarker test for gastric cancer. Mol Cancer 17: 68, 2018.

63. Yuan SX, Wang J, Yang F, Tao QF, Zhang J, Wang LL, Yang Y, Liu H, Wang ZG, Xu QG, et al: Long noncoding RNA DANCR increases stemness features of hepatocellular carcinoma by derepression of CTNNB1. Hepatology 63: 499-511, 2016.
64. Pan L, Liang W, Gu J, Zang X, Huang Z, Shi H, Chen J, Fu M, Zhang P, Xiao X, et al: Long noncoding RNA DANCR is activated by SALL4 and promotes the proliferation and invasion of gastric cancer cells. Oncotarget 9: 1915-1930, 2017.

65. Mao Z, Li H, Du B, Cui K, Xing Y, Zhao X and Zai S: LncRNA DANCR promotes migration and invasion through suppression of lncRNA-LET in gastric cancer cells. Biosci Rep 37: BSR20171070, 2017

66. Zhang X, Liang W, Liu J, Zang X, Gu J, Pan L, Shi H, Fu M, Huang Z, Zhang Y, et al: Long non-coding RNA UFC1 promotes gastric cancer progression by regulating $\mathrm{miR}-498 / \mathrm{Lin} 28 \mathrm{~b}$. J Exp Clin Cancer Res 37: 134, 2018.

67. Sanger HL, Klotz G, Riesner D, Gross HJ and Kleinschmidt AK: Viroids are single-stranded covalently closed circular RNA molecules existing as highly base-paired rod-like structures. Proc Natl Acad Sci USA 73: 3852-3856, 1976.

68. Cocquerelle C, Mascrez B, Hetuin D and Bailleul B: Mis-splicing yields circular RNA molecules. FASEB J 7: 155-160, 1993.

69. Pasman Z, Been MD and Garcia-Blanco MA: Exon circularization in mammalian nuclear extracts. RNA 2: 603-610, 1996.

70. Hansen TB, Jensen TI, Clausen BH, Bramsen JB, Finsen B, Damgaard CK and Kjems J: Natural RNA circles function as efficient microRNA sponges. Nature 495: 384-388, 2013.

71. Shao Y, Tao X, Lu R, Zhang H, Ge J, Xiao B, Ye G and Guo J: Hsa_circ_0065149 is an indicator for Early Gastric cancer screening and prognosis prediction. Pathol Oncol Res: Aug 20, 2019 (Epub ahead of print).

72. Hong Y, Qin H, Li Y, Zhang Y, Zhuang X, Liu L, Lu K, Li L, Deng X, Liu F, Shi S and Liu G: FNDC3B circular RNA promotes the migration and invasion of gastric cancer cells via the regulation of E-cadherin and CD44 expression. J Cell Physiol 234: 19895-19910, 2019.

73. Wei J, Wang J, Gao X and Qi F: Identification of differentially expressed circRNAs and a novel hsa_circ_0000144 that promote tumor growth in gastric cancer. Cancer Cell Int 19: 268, 2019.

74. Wang Y, Xu S, Chen Y, Zheng X, Li T and Guo J: Identification of hsa_circ_0005654 as a new early biomarker of gastric cancer. Cancer Biomark 26: 403-410, 2019.

75. Mosca E, Barcella M, Alfieri R, Bevilacqua A, Canti G and Milanesi L: Systems biology of the metabolic network regulated by the Akt pathway. Biotechnol Adv 30: 131-141, 2012.

76. Giguere V: Canonical signaling and nuclear activity of mTORa teamwork effort to regulate metabolism and cell growth. FEBS J 285: 1572-1588, 2018.

77. Heras-Sandoval D, Perez-Rojas JM, Hernandez-Damian J and Pedraza-Chaverri J: The role of PI3K/AKT/mTOR pathway in the modulation of autophagy and the clearance of protein aggregates in neurodegeneration. Cell Signal 26: 2694-2701, 2014.

78. Yang Y, Gao M, Lin Z, Chen L, Jin Y, Zhu G, Wang Y and Jin T: DEK promoted EMT and angiogenesis through regulating $\mathrm{PI} 3 \mathrm{~K} / \mathrm{AKT} / \mathrm{mTOR}$ pathway in triple-negative breast cancer. Oncotarget 8: 98708-98722, 2017.

79. Wu ZH, Lin C, Liu CC, Jiang WW, Huang MZ, Liu X and Guo WJ: MiR-616-3p promotes angiogenesis and EMT in gastric cancer via the PTEN/AKT/mTOR pathway. Biochem Biophys Res Commun 501: 1068-1073, 2018.

80. Zhang X, Wang S, Wang H, Cao J, Huang X, Chen Z, Xu P, Sun G, Xu J, Lv J and Xu Z: Circular RNA circNRIP1 acts as a microRNA-149-5p sponge to promote gastric cancer progression via the AKT1/mTOR pathway. Mol Cancer 18: 20, 2019.

81. Allemani C, Weir HK, Carreira H, Harewood R, Spika D, Wang XS, Bannon F, Ahn JV, Johnson CJ, Bonaventure A, et al: Global surveillance of cancer survival 1995-2009: Analysis of individual data for 25676887 patients from 279 population-based registries in 67 countries (CONCORD-2). Lancet 385: 977-1010, 2015.

82. Ren J, Zhou Q, Li H, Li J, Pang L, Su L, Gu Q, Zhu Z and Liu B: Characterization of exosomal RNAs derived from human gastric cancer cells by deep sequencing. Tumor Biol 39: 1010428317695012, 2017. 